\title{
Induction of dermal-epidermal separation in mice by passive transfer of antibodies specific to type VII collagen
}

\author{
Cassian Sitaru, ${ }^{1}$ Sidonia Mihai, ${ }^{1}$ Christoph Otto, ${ }^{2}$ Mircea T. Chiriac, ${ }^{3}$ Ingrid Hausser, ${ }^{4}$ \\ Barbara Dotterweich, ${ }^{3}$ Hitoshi Saito, ${ }^{5}$ Christian Rose, ${ }^{1}$ Akira Ishiko, ${ }^{5}$ and Detlef Zillikens ${ }^{1}$ \\ 1Department of Dermatology, University of Lübeck, Lübeck, Germany. 2Unit of Experimental Transplantation Immunology, \\ Department of Surgery, and ${ }^{3}$ Department of Dermatology, University of Würzburg, Würzburg, Germany. ${ }^{4}$ Department of Dermatology, \\ University of Heidelberg, Heidelberg, Germany. ${ }^{5}$ Department of Dermatology, Keio University School of Medicine, Tokyo, Japan.
}

\begin{abstract}
Epidermolysis bullosa acquisita (EBA) is a subepidermal blistering disorder associated with tissue-bound and circulating autoantibodies specific to type VII collagen, a major constituent of the dermal-epidermal junction. Previous attempts to transfer the disease by injection of patient autoantibodies into mice have been unsuccessful. To study the pathogenic relevance of antibodies specific to type VII collagen in vivo, we generated and characterized rabbit antibodies specific to a murine form of this antigen and passively transferred them into adult nude, $\mathrm{BALB} / \mathrm{c}$, and $\mathrm{C} 57 \mathrm{BL} / 6$ mice. Immune rabbit IgG bound to the lamina densa of murine skin and immunoblotted type VII collagen. Mice injected with purified IgG specific to type VII collagen, in contrast to control mice, developed subepidermal skin blisters, reproducing the human disease at the clinical, histological, electron microscopical, and immunopathological levels. Titers of rabbit IgG in the serum of mice correlated with the extent of the disease. $\mathrm{F}\left(\mathrm{ab}^{\prime}\right)_{2}$ fragments of rabbit IgG specific to type VII collagen were not pathogenic. When injected into C5-deficient mice, antibodies specific to type VII collagen failed to induce the disease, whereas C5-sufficient mice were susceptible to blister induction. This animal model for EBA should facilitate further dissection of the pathogenesis of this disease and development of new therapeutic strategies.
\end{abstract}

\section{Introduction}

Autoimmunity is a common event, but aberrations in this phenomenon may result in autoimmune diseases. Criteria to classify a disease as autoimmune include direct evidence from passive transfer of pathogenic antibody or pathogenic T cells into animals, indirect evidence of the reproduction of the autoimmune disease in animals by active immunization, and circumstantial evidence from clinical observations (1).

Epidermolysis bullosa acquisita (EBA), a severe chronic subepidermal blistering disease of skin and mucous membranes, is characterized by tissue-bound and circulating IgG antibodies specific to the dermal-epidermal junction (DEJ) (2). Patients' serum autoantibodies bind to the 290-kDa type VII collagen, the major component of anchoring fibrils (3, 4). Epitopes recognized by the majority of EBA sera were mapped to the noncollagenous 1 (NC1) domain of type VII collagen (5-9). The essential role that type VII collagen plays in the biology of the DEJ is exemplified by inherited or targeted disruptions in the gene that encodes it, which yield a phenotype characterized by subepidermal blisters (10-17).

The presence of tissue-bound and serum autoantibodies specific to type VII collagen in patients provides circumstantial evidence that EBA is an antibody-mediated organ-specific autoimmune disease. In addition, autoantibodies specific to type VII collagen from EBA patients were shown to recruit and activate leukocytes in vitro, resulting in

Nonstandard abbreviations used: DEJ, dermal-epidermal junction; EBA, epidermolysis bullosa acquisita; GST, gluthatione-S-transferase; IF, immunofluorescence; NC1, noncollagenous 1

Conflict of interest: The authors have declared that no conflict of interest exists.

Citation for this article: J. Clin. Invest. 115:870-878 (2005)

doi:10.1172/JCI200521386 dermal-epidermal separation in cryosections of human skin (18). However, in vivo evidence to support the autoimmune nature of EBA is still lacking. Previous attempts to induce EBA by passive transfer of patients' autoantibodies into neonatal BALB/c mice $(19,20)$ were not successful. It has been suggested that the failure to reproduce EBA in mice may be related to differences between human and murine type VII collagen or to the need for prolonged interaction of antibody with the antigen in order to induce disease $(19,20)$.

In the present study, we examined the effect of antibodies specific to type VII collagen in vivo. Patient antibodies showed a reduced ability to cross-react with murine skin. Therefore, we chose an alternative strategy, originally devised by Liu et al. (21), to study the pathogenesis of bullous pemphigoid, and generated recombinant peptides of the murine type VII collagen NC1 domain, which were used to immunize rabbits. The passive transfer of IgG from these rabbits into mice of different strains resulted in a subepidermal blistering phenotype that closely mimicked the human disease. Blister induction required both the Fc portion of rabbit IgG and activation of terminal complement components. Our study shows the capacity of antibodies specific to type VII collagen to induce subepidermal blisters and provides direct evidence to classify EBA as an autoimmune condition.

\section{Results}

EBA patients' autoantibodies bind to a lesser extent to mouse skin than to buman skin. The reactivity of IgG autoantibodies from serum of 5 patients with EBA was analyzed by immunofluorescence (IF) microscopy using human and mouse skin sections. Titers of these sera ranged from 10 to 320 when human substrate was used. Two sera showed no difference in their reactivity to mouse and human skin, 1 serum was less reactive with mouse skin, and 2 sera did not bind to mouse skin at all (Supplemental Table 1; supple- 


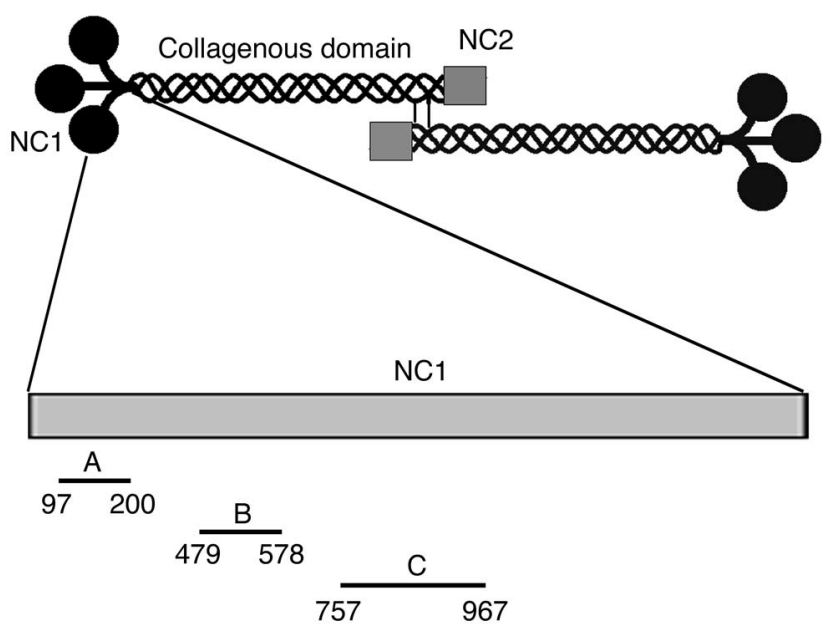

mental material available online with this article; doi:10.1172/ JCI200521386DS1). To circumvent the lack of cross-reactivity of some patient sera and to obtain larger amounts of high-titered sera with reactivity to murine type VII collagen, we immunized rabbits against recombinant forms of this antigen.

Recombinant production of type VII collagen. The cDNA sequences coding for 3 fragments of murine type VII collagen NC1 were cloned

\section{Figure 1}

Schematic organization of human type VII collagen and cDNA constructs generated for this study for expressing recombinant peptides of the NC1 domain. Type VII collagen is composed of 3 identical $\alpha$ chains, each consisting of a central triple helical collagenous domain, flanked by a large amino-terminal noncollagenous domain (NC1) and a smaller carboxy-terminal noncollagenous domain (NC2). Two molecules form antiparallel tail-to-tail dimers stabilized by disulfide bonding through a carboxy-terminal overlap between NC2 domains. Three fragments of murine type VII collagen cDNA were cloned in pGEX-6P-1 and expressed in E. coli. Amino acid residue numbers are shown below the fragments.

into a prokaryotic expression vector and expressed in Escherichia coli (Table 1 and Figure 1). The proteins, purified by glutathione-affinity chromatography, migrated consistently with their calculated masses of 38.8, 38.2, and $49.5 \mathrm{kDa}$ when separated by SDS-PAGE.

IgG from rabbit immune sera binds to the lamina densa of the epidermal basement membrane zone and recognizes type VII collagen. Circulating IgG from immune rabbit sera, but not preimmune and normal rabbit sera, bound to the DEJ, as shown by IF microscopy using murine skin as substrate at a titer of 20,480 (Figure 2, A and B). In addition, immune sera stained intact human skin (Figure $2 \mathrm{C}$ and Supplemental Table 1 ) and bound to the dermal side of $1 \mathrm{M} \mathrm{NaCl}$-split skin (data not shown). Indirect immunoelectron microscopy analysis demonstrated that rabbit
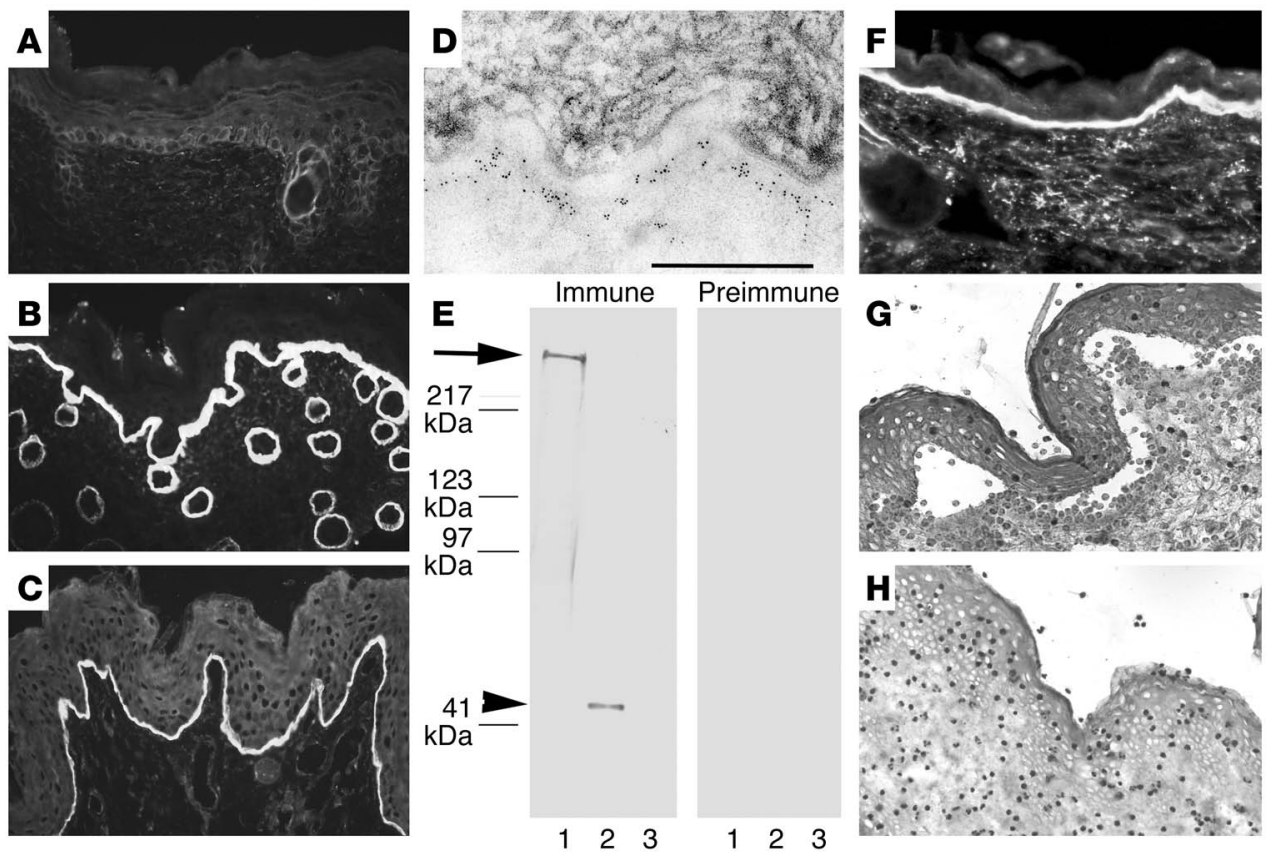

\section{Figure 2}

Serum IgG from rabbit SA2954 binds to the DEJ, recognizes type VII collagen, and activates complement and leukocytes in vitro. IF microscopy of rabbit SA9254 serum on frozen skin sections shows no specific staining before immunization (A); in contrast, after immunization with type VII collagen, IgG binds to the DEJ of mouse (B) and human (C) skin (magnification, $\times 250$ ). (D) By immunoelectron microscopy, IgG purified from immune rabbit serum binds to the lamina densa of mouse skin. Scale bar: $0.5 \mu \mathrm{m}$. (E) Extract of murine dermis (lane 1) and equimolar amounts of GST-mCOL7C (lane 2) and GST (lane 3) were separated by gradient 4-20\% SDS-PAGE and immunoblotted with immune and preimmune rabbit serum preadsorbed against GST. IgG from immune serum, but not preimmune serum, binds to both full-length cell-derived (arrow) and recombinant fragment $C$ (arrowhead) of type VII collagen. (F) Frozen murine skin sections were incubated with immune rabbit serum and subsequently with fresh serum as a source of complement. Bound murine C3 was visualized at the DEJ by FITC-labeled antibody (magnification, $\times 250$ ). When incubated with frozen sections of human skin in the presence of human leukocytes, IgG from the immune $(\mathbf{G})$, but not preimmune $(\mathbf{H})$ rabbit-induced dermalepidermal separation was observed (magnification, $\times 400$ ). 


\section{Table 1}

Primer sequences for PCR amplification of cDNA fragments of murine type VII collagen

\begin{tabular}{|c|c|c|}
\hline Fragment & Size (bp) & Primer sequences $\left(5^{\prime}-3^{\prime}\right)$ \\
\hline mCOL7A & 309 & $\begin{array}{l}\text { F: GATCGGATCCATAGAAGGAAGACTTGGCTCTGGGAGTGACAC } \\
\text { R: GATCGTCGACTCAATTGACGAAGAAGAAGAAATCG }\end{array}$ \\
\hline mCOL7B & 300 & $\begin{array}{l}\text { F: GATCGGATCCATAGAAGGAAGAACTGAGTACCGTCTCACGCTG } \\
\text { R: GATCGTCGACTCAAGACACCCGCACCGTGTA }\end{array}$ \\
\hline mCOL7C & 630 & $\begin{array}{l}\text { F: GATCGGATCCATAGAAGGAAGAGTAGCTGGTGTGGATGGAGC } \\
\text { R: GATCGTCGACTCAGTCCACCACGCGTAGTTCAG }\end{array}$ \\
\hline
\end{tabular}

$\mathrm{F}$, forward primer; $\mathrm{R}$, reverse primer. type VII collagen per injection developed single blisters on their tails and ears, often accompanied by mild erythema (Figure 3B). In mice that received $3.75 \mathrm{mg}$ of IgG specific to type VII collagen per injection $(n=4)$, initial skin lesions were observed 48-72 hours after the first injection. The blisters developed into erosions partly covered by crusts. Extensive widespread lesions were seen 4-7 days after the first injection, depending on the amount of injected IgG (Figure 3, A and F). There was no evidence of clinical lesions in mice injected with control $\operatorname{IgG}(n=4)$ at any time during the observation period (Figure 3, G-I). In all mice injected with antibodies specific to type VII antibodies labeled the lamina densa of mouse skin (Figure 2D). By immunoblot analysis, IgG antibodies from immune sera, in contrast to preimmune and normal rabbit sera, targeted both cell-derived and recombinant forms of type VII collagen (Figure 2E). Sera from immune, but not preimmune and normal rabbits, elicited deposition of murine and human $\mathrm{C} 3$ at the DEJ, as shown by IF microscopy using both murine and human skin (Figure 2F).

Rabbit antibodies specific to type VII collagen induce dermal-epidermal separation in frozen skin sections. To study the ability to induce subepidermal splits in vitro, rabbit IgG specific to type VII collagen was incubated with skin cryosections. After subsequent addition of human leukocytes, subepidermal splits developed in both human and murine skin sections incubated with antibodies specific to type VII collagen (Figure 2G). In contrast, no dermal-epidermal separation occurred in sections treated with preimmune rabbit IgG (Figure $2 \mathrm{H}$ ).

Mice injected with rabbit antibodies specific to type VII collagen develop skin blisters. Adult nude mice were injected with different doses of IgG purified from rabbits immunized with type VII collagen or from normal rabbits (see Table 2). Within 24-48 hours after the first injection, mice that received $15 \mathrm{mg}(n=4)$ and $7.5 \mathrm{mg}(n=4)$ of $\operatorname{IgG}$ specific to collagen, epidermal detachment and peeling could easily be elicited by gentle lateral mechanical pressure (Figure 3, C-E). Following the same protocol, we also administered $15 \mathrm{mg}$ of IgG specific to type VII collagen per injection to $\mathrm{BALB} / \mathrm{c}(n=2)$ and C57BL/6 $(n=2)$ mice (data not shown). Single blisters developed initially 6 days after the first injection on tail base, snout, and ears and then spread to limbs and trunk. Widespread lesions, including blisters, erosions, and crusts, were seen after 9 days in all mice injected with pathogenic IgG. $\mathrm{BALB} / \mathrm{c}(n=2)$ and C57BL/6 $(n=2)$ mice injected with normal rabbit IgG did not develop cutaneous disease (data not shown). None of the mice showed behavioral alterations. However, adult mice with skin disease showed significant weight loss (mean of $15 \%$ of initial weight, $P<0.001)$. A total of $22 \mathrm{BALB} / \mathrm{c}$ neonatal mice, which received as many as 9 daily injections of rabbit IgG specific to type VII collagen or normal rabbit IgG (10 mg IgG/g body weight per day) alone, did not develop blisters and showed no difference with regard to their weight curve. In addition, mice injected with 4 doses of the antibody specific to type VII collagen $(n=5)$ or with control antibody $(n=5)$ and then with murine neutrophils did not develop blisters. However, coinjection of neonates with neutrophils and IL-8 and C5a led to the

\section{Table 2}

Titers of rabbit antibodies correlate with the extent of disease in mice

\begin{tabular}{|c|c|c|c|c|c|c|c|c|c|c|c|}
\hline \multirow[b]{2}{*}{ Mouse } & \multirow[b]{2}{*}{$\lg G^{A}$} & \multicolumn{2}{|c|}{ Dose of IgG (mg) } & \multicolumn{4}{|c|}{ Extent of disease ${ }^{B}$} & \multicolumn{4}{|c|}{ IgG reactivityc } \\
\hline & & Per injection & Total & 0 & 4 & 8 & $12^{D}$ & 0 & 4 & 8 & 12 \\
\hline 1 & SA2953 & 15 & 90 & 0 & 2 & 3 & 4 & 0 & 320 & 1,280 & 5,120 \\
\hline 2 & SA2954 & 15 & 90 & 0 & 1 & 2 & 4 & 0 & 320 & 640 & 5,120 \\
\hline 3 & SA2953 & 15 & 90 & 0 & 2 & 2 & 4 & 0 & 320 & 640 & 5,120 \\
\hline 4 & SA2954 & 15 & 90 & 0 & 2 & 2 & 4 & 0 & 320 & 1,280 & 5,120 \\
\hline 5 & SA2953 & 7.5 & 45 & 0 & 2 & 3 & 4 & 0 & 1,280 & 1,280 & 5,120 \\
\hline 6 & SA2954 & 7.5 & 45 & 0 & 2 & 2 & 3 & 0 & 320 & 640 & 2,560 \\
\hline 7 & SA2953 & 7.5 & 45 & 0 & 2 & 3 & 4 & 0 & 640 & 1,280 & 2,560 \\
\hline 8 & SA2954 & 7.5 & 45 & 0 & 1 & 2 & 3 & 0 & 320 & 640 & 640 \\
\hline 9 & SA2953 & 3.75 & 22.5 & 0 & 1 & 1 & 2 & 0 & 160 & 160 & 1,280 \\
\hline 10 & SA2954 & 3.75 & 22.5 & 0 & 1 & 1 & 2 & 0 & 160 & 160 & 1,280 \\
\hline 11 & SA2953 & 3.75 & 22.5 & 0 & 1 & 1 & 2 & 0 & 160 & 160 & 1,280 \\
\hline 12 & SA2954 & 3.75 & 22.5 & 0 & 1 & 1 & 2 & 0 & 160 & 160 & 1,280 \\
\hline 13 & NR & 15 & 90 & 0 & 0 & 0 & 0 & 0 & 0 & 0 & 0 \\
\hline 14 & NR & 15 & 90 & 0 & 0 & 0 & 0 & 0 & 0 & 0 & 0 \\
\hline 15 & NR & 7.5 & 45 & 0 & 0 & 0 & 0 & 0 & 0 & 0 & 0 \\
\hline 16 & NR & 7.5 & 45 & 0 & 0 & 0 & 0 & 0 & 0 & 0 & 0 \\
\hline
\end{tabular}

ASource of IgG passively transferred into adult nude mice: SA2953 and SA2954, rabbits immunized with recombinant peptides of murine type VII collagen NC1; NR, normal rabbit. BExtent of disease was scored as follows: 0 , no lesions; 1 , fewer than 20 lesions or less than $10 \%$ of the skin surface; 2 , more than 20 lesions or $10-20 \%$ of the skin surface; $3,20-40 \%$ of the skin surface; $4,40-60 \%$ of the skin surface; and 5 , more than $60 \%$ of the skin surface. ${ }^{C}$ Endpoint titer of IgG reactivity to the DEJ as determined by IF microscopy on mouse skin sections. Disease activity and reactivity to the DEJ were determined before mice were injected (day 0 ) as well as 4,8 , and 12 days later. 

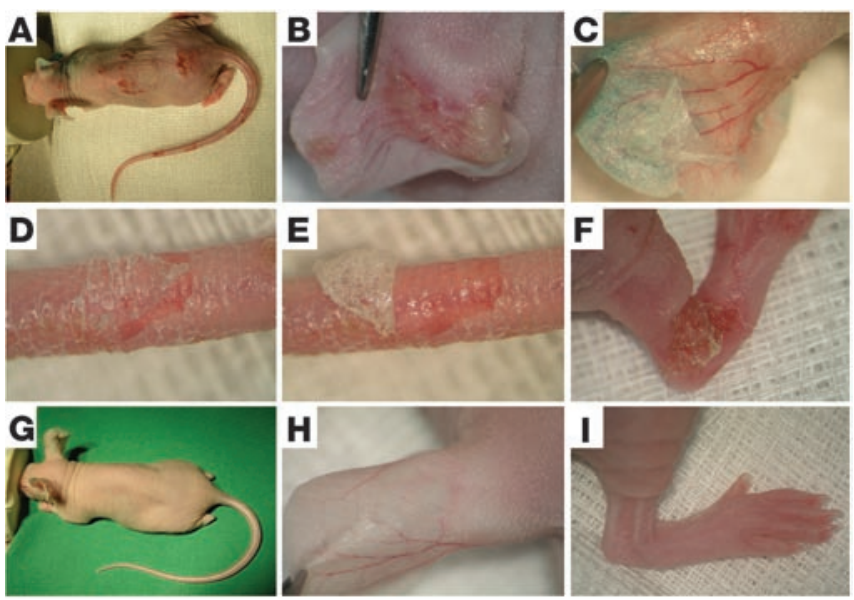

Figure 3

IgG to murine type VII collagen induces cutaneous lesions in adult mice. (A) Extensive skin lesions, including blisters, erosions, and epidermal detachment, developed in a BALB/ $\mathrm{c}^{\text {nu/nu }}$ mouse receiving, over a period of 10 days, 5 injections of $\mathrm{IgG}$, each containing $7.5 \mathrm{mg}$ of $\mathrm{IgG}$, from a rabbit immunized against murine type VII collagen. Injections into the back of the mouse induced (B) spontaneous blisters on the left ear and, upon tangential pressure, (C) epidermal detachment on the right ear. (D) Epidermal detachment was also elicited on tail skin, and (E) the epidermis could subsequently be easily lifted up from the dermis. (F) Erosions covered by crusts on the hind limb. (G-I) A control mouse challenged with the same dose of normal rabbit IgG showed no skin alterations.

development of blisters in mice treated with the pathogenic rabbit IgG specific to type VII collagen $(n=4)$, but not in mice injected with normal rabbit IgG $(n=4)$ (Supplemental Figure 1).

The blistering phenotype in mice is associated with tissue-bound immunoreactants. IF microscopy of perilesional mouse skin revealed linear deposits of rabbit IgG and murine complement $\mathrm{C} 3$ at the DEJ in adult mice that received IgG specific to type VII collagen (Figure 4, A and B). Linear deposition of rabbit IgG (Figure 4D) and murine C3 was also detected at the epithelial basement membrane of the esophagus and, less intensely, of the colon (data not shown). The relative intensity of tissue-bound immunoreactants paralleled both the extent of skin lesions and titers of serum antibodies specific to type VII collagen in diseased mice. There were no deposits of rabbit IgG and murine $\mathrm{C} 3$ at the DEJ of mice injected with control IgG (Figure 4, E and F; Supplemental Figure 1, F and H). All neonatal mice injected with rabbit antibody specific to type VII collagen demonstrated linear deposits of rabbit IgG and murine C3 at the DEJ of skin biopsies (Supplemental Figure 1, E and G), except for the mice that were killed 12 hours after the first injection, which showed no C3 deposition. Indirect IF titers of circulating rabbit IgG in neonates ranged from 320 (12 hours after the first injection) to 2,560 (after 9 injections).

Blisters induced by antibodies specific to type VII collagen localize at the sublamina densa of the basement membrane zone. From each mouse, 3 lesional skin biopsies were obtained for histopathological examination. In all adult mice injected with IgG specific to type VII collagen $(n=12)$ (Figure 5, A and B) and in all neonatal mice additionally injected with IL-8, C5a, and murine granulocytes $(n=4)$ (Supplemental Figure 1C), light microscopic analysis of skin biopsies revealed extensive dermal-epidermal separation accompanied by different degrees of inflammatory infiltrates that were dominated by neutrophils. Esophagus and colon specimens showed no histologic changes (data not shown). Histological examination of skin biopsies from adult mice injected with control IgG (Figure 5C) and from neonatal mice treated with control (Supplemental Figure 1D) and pathogenic IgG, not supplemented by neutrophils and chemoattractants, demonstrated no blisters and no inflammatory infiltrate at the DEJ. Electron microscopy of lesional skin biopsies of diseased nude mice $(n=6)$ demonstrated split formation in the uppermost dermis. The basal lamina was found in the blister roof (dermolytic blister formation) and adhered to basal keratinocytes that showed intact hemidesmosomes (Figure 6). IF analysis of lesional skin of diseased mice demonstrated IgG deposits on both the epidermal and dermal side of the cleavage (Figure 4C). By IF microscopy using autoantibodies from an EBA patient and a laminin 5-specific rabbit antibody, EBA antibodies bound to both the epidermal and dermal side, whereas rabbit antibodies specific to laminin 5 labeled only the epidermal side of the split (data not shown).

The extent of skin involvement correlates with levels of circulating serum antibodies specific to type VII collagen in diseased mice. Serum samples from adult nude mice were assayed by IF microscopy for antibody reactivity to the DEJ during the course of the disease. Samples were obtained before the first injection (day 0 ) as well as 4,8 , and 12 days later. The results of this analysis are summarized in Table 2. By intercorrelating IF microscopy titers of serum antibodies with extent of skin disease at 4 time points using the Spearman's rank correlation test, antibody reactivity strongly correlated with disease severity $(r=0.94 ; P<0.01)$. We also examined the relation between the extent of skin disease and the dose of injected IgG. Significantly more extensive disease was induced
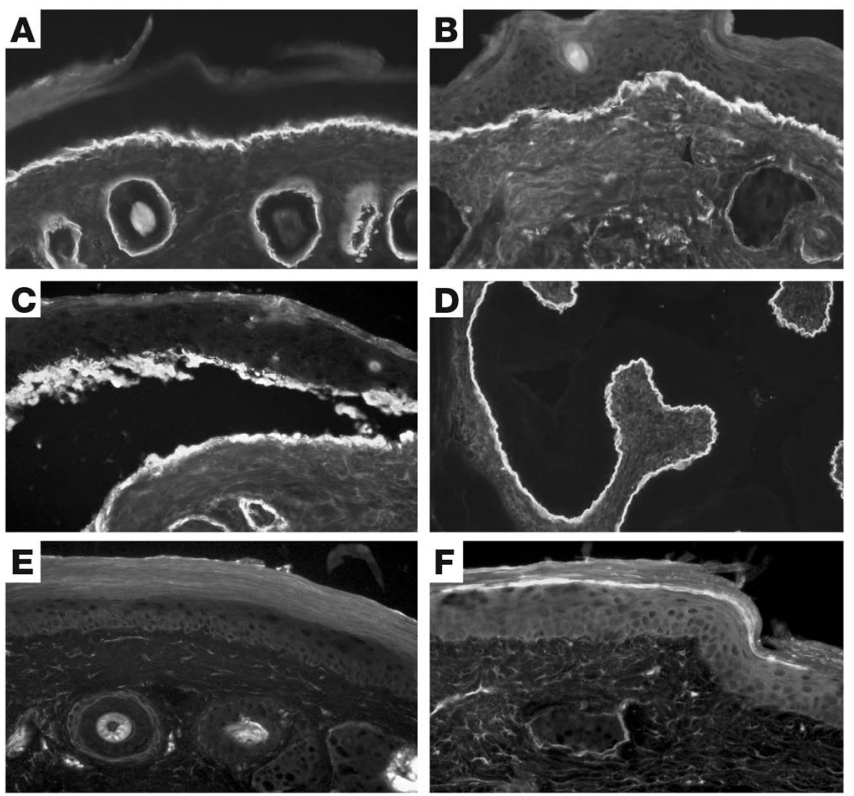

Figure 4

Rabbit IgG specific to type VII collagen binds to the basement membrane and fixes complement in vivo. IF microscopy, performed on frozen sections of a perilesional mouse skin biopsy (A and $\mathbf{B})$ and murine esophagus (D) reveals linear deposition of rabbit $\lg G(\mathbf{A}$ and $\mathbf{D})$ and murine $\mathrm{C} 3$ (B) at the basement membrane in a diseased mouse. (C) IF analysis of lesional skin from a diseased mouse shows rabbit IgG deposits on both epidermal and dermal sides of the cleavage. No deposits of rabbit $\lg G(\mathbf{E})$ or murine $\mathrm{C} 3(\mathbf{F})$ are detected at the DEJ of a mouse injected with control rabbit IgG (magnification, $\times 250$ ). 

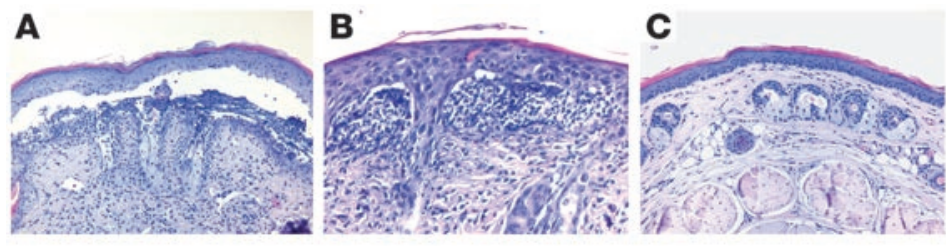

\section{Figure 5}

By light microscopy, antibodies specific to type VII collagen cause subepidermal splits. (A) Histologic examination of skin biopsies from a diseased mouse reveals extensive subepidermal cleavage (magnification, $\times 200$ ). (B) The inflammatory infiltrate is dominated by neutrophils (magnification, $\times 400$ ). (C) No specific histologic alterations are found in the skin of a mouse treated with control lgG (magnification, $\times 200$ ).

in mice injected with $7.5 \mathrm{mg}$ or $15 \mathrm{mg}$ immune IgG compared with mice treated with $3.75 \mathrm{mg} \operatorname{IgG}$ (Figure 7). Mice injected with $7.5 \mathrm{mg}$ or $15 \mathrm{mg}$ immune IgG showed no significant difference with regard to the extent of the disease.

$F\left(a b^{\prime}\right)_{2}$ fragments of pathogenic rabbit antibodies do not induce subepidermal blisters. To determine whether the Fc portion of antibodies specific to type VII collagen is of importance for blister induction in our passive transfer mouse model, we prepared $\mathrm{F}\left(\mathrm{ab}^{\prime}\right)_{2}$ fragments of rabbit IgG. In contrast to adult nude mice injected with $7.5 \mathrm{mg}$ of pathogenic rabbit IgG $(n=4)$ (Figure $8 \mathrm{~A})$, mice treated with $\mathrm{F}\left(\mathrm{ab}^{\prime}\right)_{2}$ preparations $(n=2)$, used at the same molar concentration as unfragmented IgG, failed to induce dermal-epidermal separation (Figure 8B). By IF microscopy, IgG deposits were found in perilesional skin of mice injected with intact pathogenic IgG using both antibodies to IgG-Fab (Figure 8C) and IgG-Fc (Figure 8E). In contrast, in mice receiving $\mathrm{F}\left(\mathrm{ab}^{\prime}\right)_{2}$ fragments, positive staining for Fab but not for Fc (Figure 8, D and F) or murine C3 (data not shown) was observed. By indirect IF microscopy of sera from injected mice, using a Fab-specific FITC-labeled antibody, titers of intact and fragmented rabbit antibodies were 1,280 and 2,560, respectively. Nude mice injected with intact normal rabbit IgG $(n=2)$ or with its $\mathrm{F}\left(\mathrm{ab}^{\prime}\right)_{2}$ fragments $(n=2)$ did not develop signs of cutaneous disease (data not shown).

C5-deficient mice are resistant to the induction of subepidermal blisters by antibodies specific to type VII collagen. Like C57BL/6 and BALB/c mice, C5-sufficient mice $(n=4)$ developed initial blisters 6 days after the first injection of $15 \mathrm{mg}$ of rabbit IgG specific to type VII collagen, and widespread disease was observed 9 days after the first injection (Figure 9, A and C). C5-sufficient mice treated with normal rabbit IgG $(n=2)$ did not develop skin disease. By histopathological analysis, subepidermal blisters were observed in biopsies of lesional skin (Figure 9E). Direct IF microscopy of perilesional skin showed linear deposits of rabbit IgG (Figure 9G), murine C3 (data not shown), and membrane attack complex at the DEJ (Figure 9I). In contrast, none of the C5-deficient mice treated with either pathogenic rabbit $\operatorname{IgG}(n=4)$ or normal rabbit IgG $(n=2)$ showed clinical (Figure 9, B and D) or histopathological (Figure 9F) skin lesions. IF microscopy of skin biopsies from C5-deficient mice injected with pathogenic antibody showed rabbit IgG (Figure 9H) and murine C3 (data not shown) but no membrane attack complex deposition (Figure 9J) at the DEJ. Titers of rabbit IgG specific to type VII collagen in sera of both C5-sufficient and C5-deficient mice were virtually the same, ranging from 640 to 1,280 . All mice injected with pathogenic rabbit IgG, but not those treated with control antibody, showed significant $(P<0.001)$ weight loss.

\section{Discussion}

Clinical and in vitro experimental evidence suggests that EBA is an antibody-mediated organ-specific autoimmune condition $(3,18)$. However, the disease has not yet been reproduced in animals. In this study, we show the capacity of antibodies specific to type VII collagen to induce a subepidermal bullous disease resembling EBA when passively transferred into mice.

Previous attempts to induce EBA by passive transfer of patients' autoantibodies into mice have failed $(19,20)$. In this study, we found that antibodies from EBA patients show a heterogeneous binding pattern to mouse skin; some EBA sera demonstrate a complete lack of reactivity with this substrate. Animal models of other bullous diseases, including bullous pemphigoid, pemphigus, and anti-epiligrin cicatricial pemphigoid, were developed using the passive transfer of antigen-specific antibodies from immunized rabbits (21-23). Along these lines and to circumvent the heterogeneous binding pattern of patients' IgG with murine skin, we immunized rabbits against murine type VII collagen. IgG from immunized rabbits stained the dermal side of salt-split skin, as shown by IF microscopy; bound exclusively to the lamina densa of murine skin, as shown by immunoelectron microscopy; and immunoblotted cell-derived and recombinant type VII collagen. In addition, when incubated with frozen skin sections in the presence of leukocytes, rabbit IgG, like human EBA autoantibodies (18), recruited neutrophils at the DEJ and induced dermal-epidermal separation. The ability of rabbit IgG against murine type VII collagen to cross-react with and to induce subepidermal splits in cryosections of human skin may suggest that EBA patients' autoantibodies, which cross-react with murine skin, could also be pathogenic when passively transferred into animals. However, human autoantibodies may bind to murine epitopes that are not pathogenically relevant or may not activate murine complement and/or leukocytes in a sufficient way to induce blisters. In addition, in the past, investigators may

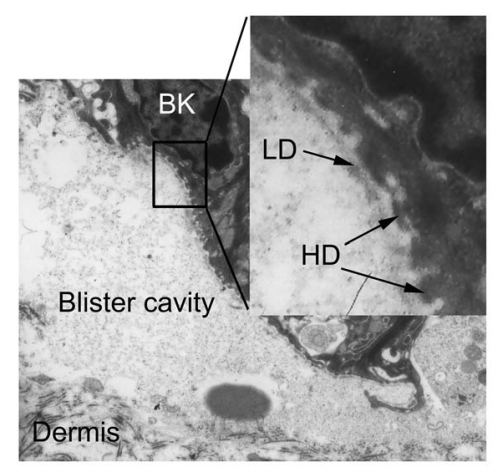

\section{Figure 6}

Experimentally induced splits localize below the lamina densa. Electron microscopic examination of a lesional skin biopsy from a diseased mouse demonstrates that the blister roof contains the lamina densa (LD) bordered by basal keratinocytes (BK) with hemidesmosomes (HD) (arrows). Dermal connective tissue represents the blister floor (magnification, $\times 11,000$; inset: magnification, $\times 44,000$ ). 


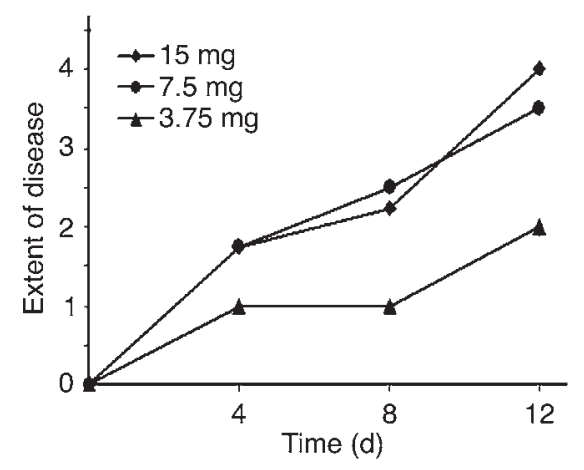

Figure 7

The extent of cutaneous disease in the mice depends on the injected amount of immune rabbit IgG. The extent of disease was scored as described in Methods and in Table 2. Means of individual clinical scores $(n=4)$ are shown before the first injection as well as 4,8 , and 12 days later. Significantly more extensive disease was induced in mice injected with $7.5 \mathrm{mg}$ or $15 \mathrm{mg}$ of immune $\mathrm{IgG}$ compared with mice treated with $3.75 \mathrm{mg}$ of IgG.

have injected human autoantibodies over too little time. Future studies with EBA sera, which must cross-react with murine skin and be available in sufficient amounts, should finally determine whether human autoantibodies can induce blisters in mice.

When injected into adult nude, BALB/c, and C57BL/6 mice, rabbit IgG specific to type VII collagen bound to the DEJ of mouse skin, activated complement, and induced subepidermal blisters. Lesions first developed in friction-exposed regions distant from the injection site, including tail and ears, and then on legs and trunk. This observation suggests that the subcutaneously injected IgG was distributed through the circulatory system and is in line with the finding of high concentrations of rabbit IgG in the peripheral blood of diseased mice throughout the observation period of 2 weeks. In addition, in agreement with the previous observation that human autoantibodies specific to type VII collagen induce dermal-epidermal separation in a dosedependent manner in an in vitro model of EBA (18), the extent of disease correlated with the titers of rabbit antibodies circulating in the serum of mice. We continued injecting mice that had already developed initial disease in order to establish a read-out system (widespread skin blistering) that is easy to interpret and reproducible. In $\mathrm{BALB} / \mathrm{c}$ and $\mathrm{C} 57 \mathrm{BL} / 6$ mice, it took longer for lesions to develop than in nude mice. This finding may be due to the abundant hair in wild-type animals that was suggested to decrease blistering in type VII collagen-deficient mice (17) and may also delay the development of blisters in normal mice injected with antibodies specific to type VII collagen. BALB/c and C57BL/ 6 strains are most commonly used for genetic manipulations. The induction of an EBA phenotype in these strains will allow the use of various knockout and transgenic mice to study the pathogenesis of blister formation in experimental EBA. In addition, the immunodeficient status of nude mice, which allows grafting of human skin and/or leukocytes, will facilitate the development of chimeric mouse/human systems.

Histological features of lesional skin in diseased mice replicated findings seen in EBA patients, including subepidermal blisters and various degrees of neutrophilic infiltrates (24). In a previous study (20), the passive transfer of high doses of purified
IgG from an EBA patient was shown to also induce an influx of granulocytes into the upper dermis of neonatal BALB/c mice, yet failed to produce blisters at the clinical or microscopic levels. We observed that the experimentally induced blisters localize below the lamina densa, which is compatible with the ultrastructural findings in the human disease (25).

During the observation period of 2 weeks, in spite of weight loss, no behavioral alterations were noted in the injected mice. In the absence of clinical or histopathological changes of oral, esophageal, and colonic mucous membranes, one may only speculate on the cause of this weight loss. Similar to various other inflammatory diseases, anorexia and an increased catabolism triggered by tissue injury may be considered as a cause for weight loss in the diseased mice (26). However, the general condition of injected mice allows them to be kept for an extended period of time. This will facilitate the continuous administration of disease-inducing antibodies to nude mice over a longer time and should be helpful for the development of new therapeutic approaches.

In the past, neonatal mice, which are hairless and of a low body weight, have been preferentially used to study the pathogenicity of antibodies in blistering skin diseases $(21,23,27)$. However, the injection of our pathogenic rabbit antibody alone did not induce blisters in newborn mice. Interestingly, in contrast to the mouse model of bullous pemphigoid $(21,28)$, we saw no infiltrates of

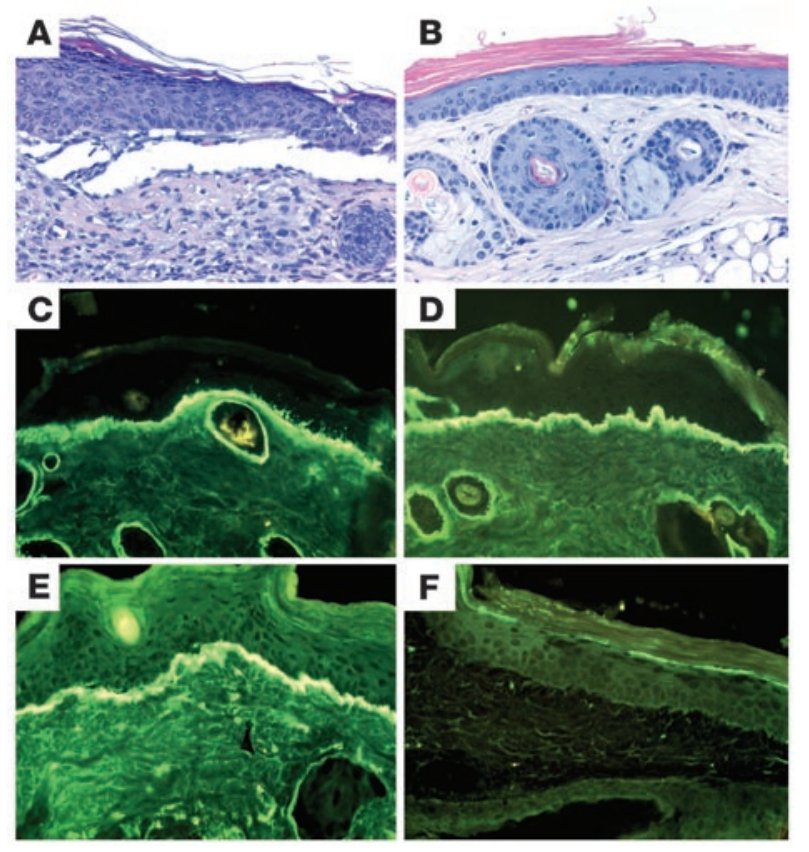

\section{Figure 8}

Dermal-epidermal separation is dependent on the Fc portion of antibodies specific to type VII collagen. (A) Subepidermal blister in a skin biopsy from a mouse receiving, over a period of 12 days, 6 injections of IgG, each containing $7.5 \mathrm{mg}$ of intact IgG from immune rabbit serum. (B) In contrast, no histological changes in a mouse injected with $F\left(a b^{\prime}\right)_{2}$ fragments generated by pepsin digestion of pathogenic rabbit IgG (H\&E, magnification, $\times 200)$. IF analysis of perilesional skin from a diseased mouse revealed positive staining using both anti-Fab (C) and anti-Fc (E) FITC-conjugated antibodies. In a mouse injected with $F\left(a b^{\prime}\right)_{2}$ fragments, staining was observed with the Fab- (D) but not with the Fc-specific (F) conjugate $(\times 400)$. 

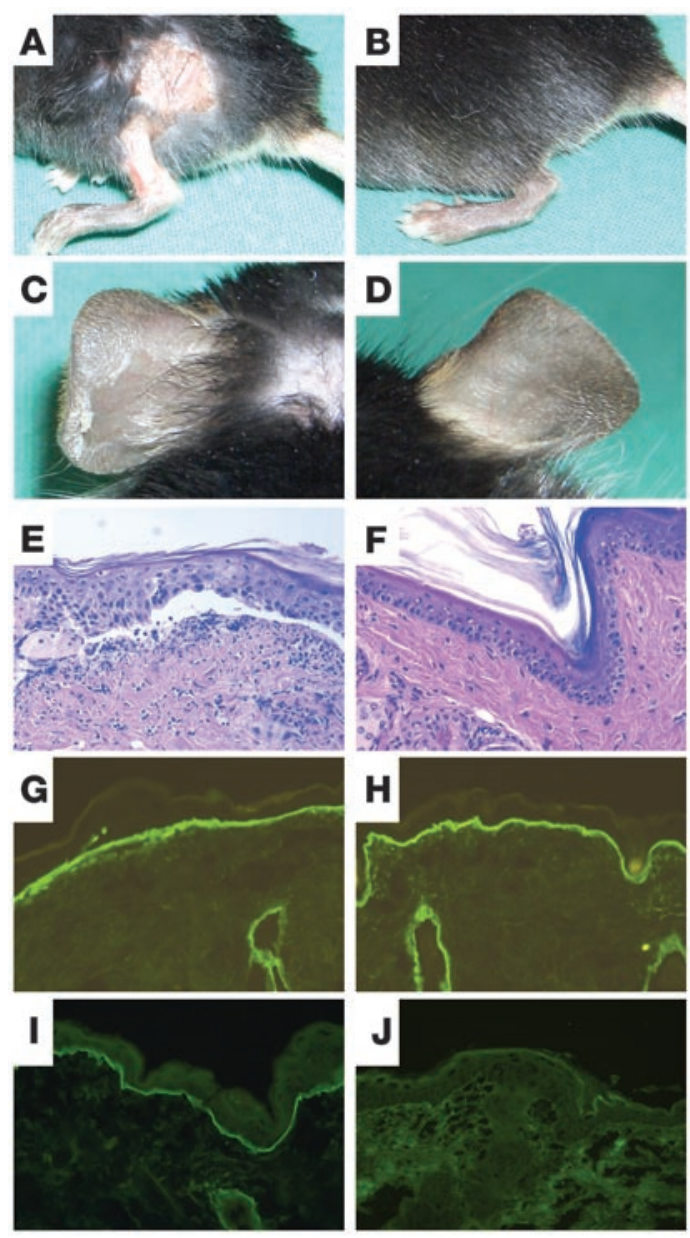

\section{Figure 9}

C5-deficient mice are resistant to the induction of cutaneous disease by antibodies specific to type VII collagen. Erosions on back and leg and, upon tangential pressure, epidermal detachment on the ear in a C5-sufficient (A and $\mathbf{C}$ ) but not in a C5-deficient (B and $\mathbf{D}$ ) mouse. Mice received 5 injections, each containing $15 \mathrm{mg}$ of $\mathrm{IgG}$ from immune rabbit serum, over a period of 10 days. Histologic analysis of murine skin revealed subepidermal cleavage and a neutrophil-rich inflammatory infiltrate in C5-sufficient mice (E) but no histological changes in C5-deficient mice (F) (magnification, $\times 200$ ). IF analysis of mouse skin showed deposition of rabbit IgG in mice both deficient $(\mathbf{G})$ and sufficient $(\mathbf{H})$ in C5. Deposits of membrane attack complex along the dermal-epidermal junction were only observed in C5-sufficient (I) and not in C5-deficient $(\mathbf{J})$ mice (magnification, $\times 400$ ).

and lectin pathways of complement activation. C5 deficiency not only eliminates the production of $\mathrm{C} 5 \mathrm{a}$ but also prevents the downstream activation of other complement components with subsequent formation of the membrane attack complex. We found C5-deficient mice resistant to the induction of blisters by antibodies specific to type VII collagen. These results demonstrate that activation of terminal complement components is required for blister formation in experimental EBA. Future studies will also address the role of leukocytes for lesion induction in this model.

In conclusion, this study demonstrates that antibodies specific to the NC1 domain of type VII collagen are pathogenic when passively transferred into mice. The blistering phenotype induced in mice recapitulates the main clinical, histological, and immunopathological features of human EBA. Blister formation requires the $\mathrm{Fc}$ portion of antibodies specific to type VII collagen and an intact complement system of injected mice. These studies establish what we believe to be the first animal model of EBA that will be a useful tool for dissecting the cellular and molecular mechanisms of blister formation in EBA. In addition, this experimental system will facilitate the development of more effective therapeutic strategies for managing this severe autoimmune disorder.

\section{Methods}

Patients. Serum samples were obtained from 5 patients with EBA prior to the initiation of treatment. Patients were characterized by (a) blisters on the skin; (b) linear deposits of IgG at the DEJ, as shown by direct IF microscopy; (c) circulating IgG autoantibodies binding to the dermal side of $1 \mathrm{~mol} / \mathrm{l}$ of NaCl-split human skin, as shown by indirect IF microscopy; and (d) immunoblot reactivity with type VII collagen extracted from dermis. For the experiments conducted, we obtained approval from the Ethics Committee of the medical faculty of the University of Würzburg, Würzburg, Germany (Institutional Board Project no. 37/98). We obtained informed consent from patients whose material was used in the study, in adherence to the Helsinki Principles.

Mice. Seven- to 8-week-old BALB/c, C57BL/6J, and BALB/c $\mathrm{c}^{\text {nu/nu }}$ female mice with a body weight of approximately $20 \mathrm{~g}$, as well as pregnant $\mathrm{BALB} / \mathrm{c}$ mice, were obtained from Charles River. C5-deficient (B10.D2$\left.H c^{0} H 2^{d} H 2-T 18^{c} / o S n J\right) ~(29)$ and C5-sufficient (B10.D2- $H c^{1} H 2^{d} H 2-T 18^{c} /$ $\mathrm{nSnJ}$ ) mice were obtained from Jackson Laboratories. All injections and bleedings were performed on mice narcotized by inhalation of isoflurane or intraperitoneal administration of a mixture of ketamine $(100 \mu \mathrm{g} / \mathrm{g})$ and xylazine $(15 \mu \mathrm{g} / \mathrm{g})$. The experiments were approved by the Animal Care and Use Committee (Kiel, Germany; no. 83/02 and 6/g/04) and performed by certified personnel. 
Cell culture. PAM 212 murine keratinocytes (30) were cultured in DMEM (CCPro) supplemented with $10 \%$ fetal calf serum, $4 \mathrm{mM}$ L-glutamine, $100 \mathrm{U}$ penicillin, and $100 \mu \mathrm{g} / \mathrm{ml}$ streptomycin (all from CCpro).

Heterologous expression of murine type VII collagen fragments. Three fragments of murine type VII collagen were expressed as glutathione-Stransferase (GST) fusion proteins (Figure 1). Primers for PCR were synthesized by MWG Biotech. DNA sequence data for murine type VII collagen was retrieved from GenBank using the accession number NM_ 007738 (31). The cDNA fragments were obtained by PCR on a cDNA pool generated by reverse transcription of total RNA from murine PAM 212 keratinocytes. Restriction sites for BamHI and SalI were introduced during PCR using the primer pairs depicted in Table 1. The reaction was run in a thermal cycler GeneAmp PCR System 9700 (Applied Biosystems) for 35 cycles of 30 seconds denaturation at $96^{\circ} \mathrm{C}, 30$ seconds annealing at $55^{\circ} \mathrm{C}, 20$ seconds extension at $72^{\circ} \mathrm{C}$, and finally 5 minutes extra extension for the last cycle. Type VII collagen cDNA fragments were cloned into linearized pGEX-6P-1 (Amersham Biosciences) resulting in the recombinant vectors pGEX-mCOL7A, pGEX-mCOL7B, and PGEX-mCOL7C. Correct ligation and in-frame insertion of the various DNA fragments were confirmed by DNA sequence analysis. Recombinant fusion proteins were expressed in E. coli BL21 and purified by glutathione-agarose affinity chromatography (32). Protein concentrations were measured spectrophotometrically at $280 \mathrm{~nm}$ (BioPhotometer).

Immunization of rabbits. Two New Zealand White rabbits (SA2953 and SA2954) were immunized subcutaneously with $200 \mu \mathrm{g}$ of an equimolar mixture of the 3 purified recombinant proteins suspended in Freund's complete adjuvant. The animals were boosted twice (at 15-day interval) with the same protein preparation in incomplete Freund's adjuvant. Immune sera were obtained at regular intervals and characterized by IF microscopy on cryosections of murine skin and by immunoblotting using cell-derived and recombinant type VII collagen. Normal rabbit serum was obtained from CCPro.

Affinity-purification of IgG. IgG from rabbit serum was isolated using Protein G Sepharose Fast Flow affinity column chromatography (Amersham Biosciences) as described (33). Antibodies were eluted with $0.1 \mathrm{M}$ glycine buffer ( $\mathrm{pH} 2.5$ ), neutralized with $1.5 \mathrm{M}$ Tris- $\mathrm{HCl}$ ( $\mathrm{pH} 10)$, and concentrated under extensive washing with PBS ( $\mathrm{pH} 7.2$ ) using Ultrafree 15 filters (Millipore). Purified IgG was filter-sterilized (pore size, $0.22 \mu \mathrm{m}$; Millipore) and the protein concentration was measured spectrophotometrically at $280 \mathrm{~nm}$. Reactivity of IgG fractions was analyzed by IF microscopy on murine skin and by immunoblotting with cell-derived or recombinant type VII collagen. The split-inducing capacity of purified rabbit antibodies was evaluated using an in vitro assay as reported (18).

Preparation of $\mathrm{F}\left(a b^{\prime}\right)_{2}$ fragments. Preparation of $\mathrm{F}\left(\mathrm{ab}^{\prime}\right)_{2}$ fragments by pepsin digestion of rabbit IgG was performed as described $(18,34)$. By unreduced SDS-PAGE, $\mathrm{F}\left(\mathrm{ab}^{\prime}\right)_{2}$ fragments migrated at $110 \mathrm{kDa}$. Completeness of fragmentation and titers of $\mathrm{F}\left(\mathrm{ab}^{\prime}\right)_{2}$ preparations were tested by indirect IF microscopy on murine skin sections using FITC-labeled secondary antibodies specific to Fab and Fc portions of rabbit IgG, respectively (both ACRIS Antibodies $\mathrm{GmbH}$ ).

Immunofluorescence microscopy and immunoblot analysis. Complementfixing activity of antibodies to the DEJ was determined as described (35). Briefly, sections of mouse and human skin were incubated with rabbit IgG and subsequently with fresh mouse serum as a source of complement. C3 deposits were visualized using a specific FITC-labeled secondary antibody (Cappel Organon-Teknika). Frozen sections were prepared from tissue biopsies and analyzed by IF microscopy using 100fold diluted antibodies specific to rabbit IgG (DAKOCytomation) and to murine $\mathrm{C} 3$. To detect membrane attack complex deposits in murine skin, a 1:100 dilution of a rabbit antibody to human membrane attack complex (Merck) was used (36); components of the human and mouse membrane attack complex are known to be highly homologous (37). Rabbit IgG to membrane attack complex and to laminin 5 (kind gift of Kim Yancey, Medical College of Wisconsin, Milwaukee, Wisconsin, USA) were labeled with Alexa 488-conjugated goat anti-rabbit Fab using a Zenon labeling kit (Molecular Probes). Extracts of murine dermis were prepared as described (38). Recombinant proteins or dermal extracts were fractionated by $12 \%$ and $6 \%$ SDS-PAGE, respectively, transferred to nitrocellulose, and analyzed by immunoblotting (18). Alternatively, proteins were separated by gradient 4-20\% SDS-PAGE. Immunoadsorption of rabbit serum with cell lysate of bacteria transformed with wild-type pGEX was performed as described (32).

Passive transfer studies and evaluation of mice. Purified rabbit IgG specific to murine type VII collagen or normal rabbit IgG (both used at a concentration of $15 \mathrm{mg} / \mathrm{ml}$ ) was injected subcutaneously into the backs of adult mice every second day over a period of 12 days (different amounts of injected IgG are shown in Table 2). Neonatal mice (24-36 hours of age) were injected with $10 \mathrm{mg}$ rabbit IgG per g body weight every 24 hours over a period of 9 days, while neonates were kept with the mother. Recombinant human IL-8 (Merck) and C5a (Sigma-Aldrich) were prepared and administered as described $(28,39)$. Purification of granulocytes from mice followed published protocols $(40,41)$, and $5 \times 10^{6}$ granulocytes were injected subcutaneously. To avoid injection of excessive fluid volumes, rabbit antibodies were concentrated to $100 \mathrm{mg} / \mathrm{ml}$. Daily, mice were weighed and examined for their general condition and for evidence of cutaneous lesions (i.e., erythema, blisters, erosions, and crusts). Intact blisters or erosions were counted and the extent of skin disease was scored as follows: 0 , no lesions; 1 , fewer than 20 lesions or less than $10 \%$ of the skin surface; 2 , more than 20 lesions or $10-20 \%$ of the skin surface; $3,20-40 \%$ of the skin surface; $4,40-60 \%$ of the skin surface; and 5 , more than $60 \%$ of the skin surface.

To evaluate the correlation of antibody titers with the extent of disease, sera were obtained from adult nude mice at 4 different time points (Table 2). For the remaining experiments in adult mice, serum samples were collected 2 days after the last injection. Sera were assayed for antibody titers by indirect IF microscopy on cryosections of mouse skin. Biopsies of lesional and perilesional skin, esophagus, and colon were obtained 2 days after the last injection and prepared for examination by histopathology, electron microscopy, and IF microscopy. Neonatal mice were evaluated at days 0.5 $(n=4), 1(n=4), 2(n=4), 3(n=4), 4(n=2), 6(n=2)$, and $9(n=2)$ by histopathology and by both direct and indirect IF microscopy.

Histological and electron microscopical studies. Biopsies of lesional and perilesional skin, oral mucosa, esophagus, and colon were fixed in $4 \%$ buffered formalin. Sections from paraffin-embedded tissues were stained with H\&E. For electron microscopy, specimens collected from 6 diseased mice and 1 control mouse were fixed in $3 \%$ glutaraldehyde solution in $0.1 \mathrm{M}$ cacodylate buffer, $\mathrm{pH} 7.4$, for at least 2 hours at room temperature, cut into pieces of $1 \mathrm{~mm}^{3}$, washed in $0.1 \mathrm{M}$ cacodylate buffer, postfixed in $1 \%$ osmium tetroxide for 1 hour at $4{ }^{\circ} \mathrm{C}$, rinsed in water, dehydrated in graded ethanol solutions, transferred in propylene oxide, and embedded in epoxy resin (42). Before examination, ultrathin sections were treated with uranyl acetate and lead citrate. To characterize the binding site of anti-type VII collagen antibodies ultrastructurally, postembedding immunoelectron microscopy using normal mouse skin was performed as described (43). In brief, normal murine nasal skin was cryofixed with liquid propane cooled at $-190^{\circ} \mathrm{C}$, freeze-substituted with methanol, and embedded in Lowicryl $\mathrm{K} 11 \mathrm{M}$ (Chemische Werke Lowi). Ultrathin sections were incubated with anti-type VII collagen antibodies followed by $5 \mathrm{~nm}$ gold-conjugated goat anti-rabbit IgG (Amersham Biosciences). Gold labeling was enhanced in size with the InteSE silver enhancement kit (Amersham Biosciences). 
Statistical analysis. To compare the weights of diseased and control mice, the independent samples Student's $t$ test was used. Differences in disease severity were calculated using the $\chi^{2}$ test. To estimate the correlation between antibody reactivity to the DEJ, as detected by indirect IF microscopy, and disease activity, the Spearman's rank correlation test was applied (44).

\section{Acknowledgments}

This work was supported by grant Zi 439/6-1 (to D. Zillikens) from the Deutsche Forschungsgemeinschaft. We thank Eva-Bettina Bröcker and Karin Ulrichs (University of Würzburg) and
Arno Kromminga (University of Hamburg) for helpful advice and Franziska Müller (University of Lübeck) for technical assistance.

Received for publication February 20, 2004, and accepted in revised form December 21, 2004.

Address correspondence to: Cassian Sitaru, Department of Dermatology, University of Lübeck, Ratzeburger Allee 160, 23538 Lübeck, Germany. Phone: 49-451-500-2530; Fax: 49-451-500-2981; E-mail: csitaru@fastmail.fm.
1. Rose, N.R., and Bona, C. 1993. Defining criteria for autoimmune diseases (Witebsky's postulates revisited). Immunol. Today. 14:426-430.

2. Schmidt, E., and Zillikens, D. 2000. Autoimmune and inherited subepidermal blistering diseases: advances in the clinic and the laboratory. $A d v$. Dermatol. 16:113-157.

3. Woodley, D.T., et al. 1984. Identification of the skin basement-membrane autoantigen in epidermolysis bullosa acquisita. N. Engl. J. Med. 310:1007-1013.

4. Stanley, J.R., Rubinstein, N., and Klaus-Kovtun, V. 1985. Epidermolysis bullosa acquisita antigen is synthesized by both human keratinocytes and human dermal fibroblasts. J. Invest. Dermatol. 85:542-545.

5. Woodley, D.T., et al. 1988. Epidermolysis bullosa acquisita antigen is the globular carboxyl terminus of type VII procollagen. J. Clin. Invest. 81:683-687.

6. Lapiere, J.C., et al. 1993. Epitope mapping of type VII collagen. Identification of discrete peptide sequences recognized by sera from patients with acquired epidermolysis bullosa. J. Clin. Invest. 92:1831-1839.

7. Gammon, W.R., et al. 1993. Autoantibodies to type VII collagen recognize epitopes in a fibronectinlike region of the noncollagenous (NC1) domain. J. Invest. Dermatol. 100:618-622.

8. Tanaka, T., Furukawa, F., and Imamura, S. 1994. Epitope mapping for epidermolysis bullosa acquisita autoantibody by molecularly cloned cDNA for type VII collagen. J. Invest. Dermatol. 102:706-709.

9. Chen, M., Keene, D.R., Costa, A.M., Tahk, S.H., and Woodley, D.T. 2001. The carboxy-terminus of type VII collagen mediates antiparallel dimer formation and constitutes a new antigenic epitope for epidermolysis bullosa acquisita autoantibodies. J. Biol. Chem. 276:21649-21655.

10. Leigh, I.M., et al. 1988. Type VII collagen is a normal component of epidermal basement membrane, which shows altered expression in recessive dystrophic epidermolysis bullosa. J. Invest. Dermatol. 90:639-642.

11. Bruckner-Tuderman, L., Mitsuhashi, Y., Schnyder, U.W., and Bruckner, P. 1989. Anchoring fibrils and type VII collagen are absent from skin in severe recessive dystrophic epidermolysis bullosa. J. Invest. Dermatol. 93:3-9.

12. Bruckner-Tuderman, L., Niemi, K.M., Kero, M., Schnyder, U.W., and Reunala, T. 1990. Type VII collagen is expressed but anchoring fibrils are defective in dystrophic epidermolysis bullosa inversa. $\mathrm{Br} . \mathrm{J}$. Dermatol. 122:383-390.

13. Smith, L.T., and Sybert, V.P. 1990. Intra-epidermal retention of type VII collagen in a patient with recessive dystrophic epidermolysis bullosa. J. Invest. Dermatol. 94:261-264.

14. Christiano, A.M., et al. 1993. A missense mutation in type VII collagen in two affected siblings with recessive dystrophic epidermolysis bullosa. Nat. Genet. 4:62-66

15. Christiano, A.M., Ryynanen, M., and Uitto, J. 1994. Dominant dystrophic epidermolysis bullosa: identification of a Gly-- >Ser substitution in the triplehelical domain of type VII collagen. Proc. Natl. Acad. Sci. U. S. A. 91:3549-3553.

16. Hovnanian, A., et al. 1997. Characterization of 18 new mutations in COL7A1 in recessive dystrophic epidermolysis bullosa provides evidence for distinct molecular mechanisms underlying defective anchoring fibril formation. Am. J. Hum. Genet. 61:599-610.

17. Heinonen, S., et al. 1999. Targeted inactivation of the type VII collagen gene (Col7a 1$)$ in mice results in severe blistering phenotype: a model for recessive dystrophic epidermolysis bullosa. J. Cell Sci. 112:3641-3648

18. Sitaru, C., Kromminga, A., Hashimoto, T., Bröcker, E.B., and Zillikens, D. 2002. Autoantibodies to type VII collagen mediate Fcgamma-dependent granulocyte activation and induce dermal-epidermal separation in cryosections of human skin. Am. J. Pathol. 161:301-311.

19. Shigemoto, T., Nashiro, K., Tsuchida, T., Seki, Y., and Tamaki, K. 1988. Administration of IgG fraction of epidermolysis bullosa acquisita (EBA) serum into mice. J. Dermatol. 15:123-127.

20. Borradori, L., et al. 1995. Passive transfer of autoantibodies from a patient with mutilating epidermolysis bullosa acquisita induces specific alterations in the skin of neonatal mice. Arch. Dermatol. 131:590-595.

21. Liu, Z., et al. 1993. A passive transfer model of the organ-specific autoimmune disease, bullous pemphigoid, using antibodies generated against the hemidesmosomal antigen, BP180. J. Clin. Invest. 92:2480-2488

22. Memar, O., et al. 1996. Induction of blister-causing antibodies by a recombinant full-length, but not the extracellular, domain of the pemphigus vulgaris antigen (desmoglein 3). J. Immunol. 157:3171-3177.

23. Lazarova, Z., Yee, C., Darling, T., Briggaman, R.A., and Yancey, K.B. 1996. Passive transfer of anti-laminin 5 antibodies induces subepidermal blisters in neonatal mice. J. Clin. Invest. 98:1509-1518.

24. Hallel-Halevy, D., Nadelman, C., Chen, M., and Woodley, D.T. 2001. Epidermolysis bullosa acquisita: update and review. Clin. Dermatol. 19:712-718.

25. Nieboer, C., Boorsma, D.M., Woerdeman, M.J., and Kalsbeek, G.L. 1980. Epidermolysis bullosa acquisita. Immunofluorescence, electron microscopic and immunoelectron microscopic studies in four patients. Br. J. Dermatol. 102:383-392.

26. Guirao, X. 2002. Impact of the inflammatory reaction on intermediary metabolism and nutrition status. Nutrition. 18:949-952.

27. Anhalt, G.J., Labib, R.S., Voorhees, J.J., Beals, T.F., and Diaz, L.A. 1982. Induction of pemphigus in neonatal mice by passive transfer of IgG from patients with the disease. N. Engl. J. Med. 306:1189-1196.

28. Liu, Z., et al. 1997. A major role for neutrophils in experimental bullous pemphigoid. J. Clin. Invest. 100:1256-1263.

29. Nilsson, U.R., and Muller-Eberhard, H.J. 1967. Deficiency of the fifth component of complement in mice with an inherited complement defect. J. Exp. Med. 125:1-16.

30. Yuspa, S.H., Hawley-Nelson, P., Koehler, B., and Stanley, J.R. 1980. A survey of transformation markers in differentiating epidermal cell lines in culture. Cancer Res. 40:4694-4703.

31. Li, K., et al. 1993. cDNA cloning and chromosomal mapping of the mouse type VII collagen gene (Col7a1): evidence for rapid evolutionary divergence of the gene. Genomics. 16:733-739.

32. Zillikens, D., et al. 1997. Tight clustering of extracellular BP180 epitopes recognized by bullous pemphigoid autoantibodies. J. Invest. Dermatol. 109:573-579.

33. Sitaru, C., et al. 2002. Autoantibodies to bullous pemphigoid antigen 180 induce dermal-epidermal separation in cryosections of human skin. J. Invest. Dermatol. 118:664-671.

34. Liu, Z., et al. 1995. The role of complement in experimental bullous pemphigoid. J. Clin. Invest. 95:1539-1544.

35. Sitaru, C., et al. 2003. Pemphigoid gestationis: maternal sera recognise epitopes restricted to the $\mathrm{N}$-terminal portion of the extracellular domain of BP180 not present on its shed ectodomain. Br. J. Dermatol. 149:420-422.

36. Tuzun, E., Scott, B.G., Goluszko, E., Higgs, S., and Christadoss, P. 2003. Genetic evidence for involvement of classical complement pathway in induction of experimental autoimmune myasthenia gravis. J. Immunol. 171:3847-3854.

37. Mondragon-Palomino, M., Pinero, D., NicholsonWeller, A., and Laclette, J.P. 1999. Phylogenetic analysis of the homologous proteins of the terminal complement complex supports the emergence of C6 and C7 followed by C8 and C9. J. Mol. Evol. 49:282-289.

38. Shimanovich, I., et al. 2003. The autoantigen of anti-p200 pemphigoid is an acidic noncollagenous $\mathrm{N}$-linked glycoprotein of the cutaneous basement membrane. J. Invest. Dermatol. 121:1402-1408.

39. Zillikens, D., et al. 2001. Antibodies to desmogleins 1 and 3, but not to BP180, induce blisters in human skin grafted onto SCID mice. J. Pathol. 193:117-124

40. Liu, Z., et al. 2000. A critical role for neutrophil elastase in experimental bullous pemphigoid. J. Clin. Invest. 105:113-123.

41. Shimanovich, I., et al. 2004. Granulocyte-derived elastase and gelatinase B are required for dermalepidermal separation induced by autoantibodies from patients with epidermolysis bullosa acquisita and bullous pemphigoid. J. Pathol. 204:519-527.

42. Hausser, I., Fartasch, M., Schleiermacher, E., and Anton-Lamprecht, I. 1987. Disseminated cicatricial pemphigoid in a child and in an adult. Ultrastructural diagnostic criteria and differential diagnosis with special reference to acquired epidermolysis bullosa. Arch. Dermatol. Res. 279:357-365.

43. Ishiko, A., et al. 1993. Human autoantibodies against the $230-\mathrm{kD}$ bullous pemphigoid antigen (BPAG1) bind only to the intracellular domain of the hemidesmosome, whereas those against the $180-\mathrm{kD}$ bullous pemphigoid antigen (BPAG2) bind along the plasma membrane of the hemidesmosome in normal human and swine skin. J. Clin. Invest. 91:1608-1615.

44. Sitaru, C., et al. 2004. Immunoblotting and enzyme-linked immunosorbent assay for the diagnosis of pemphigoid gestationis. Obstet. Gynecol. 103:757-763. 